\title{
Blasting through lattice calculations using CUDA
}

\section{Kipton Barros}

Department of Physics, Boston University, Boston, MA 02215

E-mail: kbarros@bu.edu

\section{Ronald Babich}

Department of Physics, Boston University, Boston, MA 02215

E-mail: rbabich@bu.edu

\section{Richard Brower}

Department of Physics, Boston University, Boston, MA 02215

E-mail: browerabu .edu

\section{Michael A. Clark}

Center for Computational Science, Boston University, Boston, MA 02215

E-mail: mikec@bu.edu

\section{Claudio Rebbi*}

Department of Physics, Boston University, Boston, MA 02215

E-mail: rebbiabu.edu

\begin{abstract}
Modern graphics hardware is designed for highly parallel numerical tasks and provides significant cost and performance benefits. Graphics hardware vendors are now making available development tools to support general purpose high performance computing. Nvidia's CUDA platform, in particular, offers direct access to graphics hardware through a programming language similar to $\mathrm{C}$. Using the CUDA platform we have implemented a Wilson-Dirac operator which runs at an effective 68 Gflops on the Tesla C870. The recently released GeForce GTX 280 runs this same code at 92 Gflops, and we expect further improvement pending code optimization.
\end{abstract}

The XXVI International Symposium on Lattice Field Theory

July 14 - 19, 2008

Williamsburg, Virginia, USA

\footnotetext{
* Speaker.
} 


\section{Introduction}

For decades, Moore's law has reliably given a doubling of the number of transistors per chip about every two years, a trend that continues to this day. In the past, such increases translated directly into improved performance for serial code through higher clock rates, larger caches, and increased exploitation of instruction-level parallelism. Recently, however, such improvements have yielded diminishing returns, bringing us to the era of multi-core CPUs. For the intrinsically parallel tasks commonly found in scientific computing, this is a welcome development. Still, it is not obvious that commodity processors, whose high clock rates and large caches come at the expense of greater numbers of cores, represent the optimal balance for highly parallel workloads. Graphics processing units (GPUs), driven by the enormous video game market, represent a different set of trade-offs. GPUs emphasize very high parallelism and memory bandwidth, a recipe for astounding performance in many scientific applications.

In lattice gauge theory (LGT), the application of the Wilson-Dirac operator is a performance critical task and has been shown to map well onto GPU architectures [1. 2]. We describe an implementation of the Wilson-Dirac operator which runs at an effective 92 Gflops on the Nvidia GTX 280 GPU. Our implementation uses Nvidia's CUDA (Compute Unified Device Architecture) platform.

CUDA is a C-like programming language and a full software development toolkit [3]. CUDA provides direct and relatively low-level access to the GPU. Current high-end Nvidia graphics cards are specifically designed to facilitate general purpose computation through CUDA. An important feature, which is available in CUDA but not previous frameworks, is high speed synchronization and data sharing between threads.

Besides CUDA, several other platforms exist for highly threaded computation. Advanced Micro Devices (AMD) is another leading vendor of GPUs and has introduced the Stream SDK toolkit [ [ $₫$ ]. The Cell processor, originally designed by Sony, Toshiba, and IBM for consumer applications, is also an attractive target for LGT [5, 6, 7]. Even among mainstream CPUs there is a trend toward increasing numbers of parallel cores. For example, Intel's Larrabee architecture is expected to have tens of cores, blurring the distinction between CPU and GPU architectures [8]. A potential advantage of Nvidia's CUDA platform is that it may be used to target both GPUs and multi-core CPUs. Finally, we note that the proposed OpenCL standard [9] describes a programming model quite similar to CUDA.

\section{Hardware}

Nvidia produces three lines of graphics cards. The GeForce series serves the lucrative consumer video game market, while the Tesla series targets the high performance computing (HPC) market. Tesla cards retain the core architecture of the consumer cards but offer more device memory and greater reliability, at the expense of lower memory bandwidth and increased cost. ${ }^{1}$ Finally, Nvidia markets the Quadro line for professional graphics applications.

To date, there have been roughly two generations of CUDA-enabled GPUs. The flagship consumer cards of the previous and current generation are the GeForce 8800 GTX and the GTX

\footnotetext{
${ }^{1}$ The Tesla series also lacks video output.
} 


\begin{tabular}{|l|l|l|l|l|}
\hline Card & Cores & Bandwidth (GB/s) & Gflops & Device Memory \\
\hline GeForce 8800 GTX & 128 & 86.4 & 518 & $768 \mathrm{MB}$ \\
Tesla C870 & 128 & 76.8 & 518 & $1.5 \mathrm{~GB}$ \\
GeForce GTX 280 & 240 & 141.7 & 933 & $1 \mathrm{~GB}$ \\
Tesla C1060 & 240 & 102 & 933 & $4 \mathrm{~GB}$ \\
\hline
\end{tabular}

Table 1: Specifications of representative Nvidia cards. The Tesla S1070 (not listed) is a 1U unit, containing the equivalent of four Tesla C1060 cards.

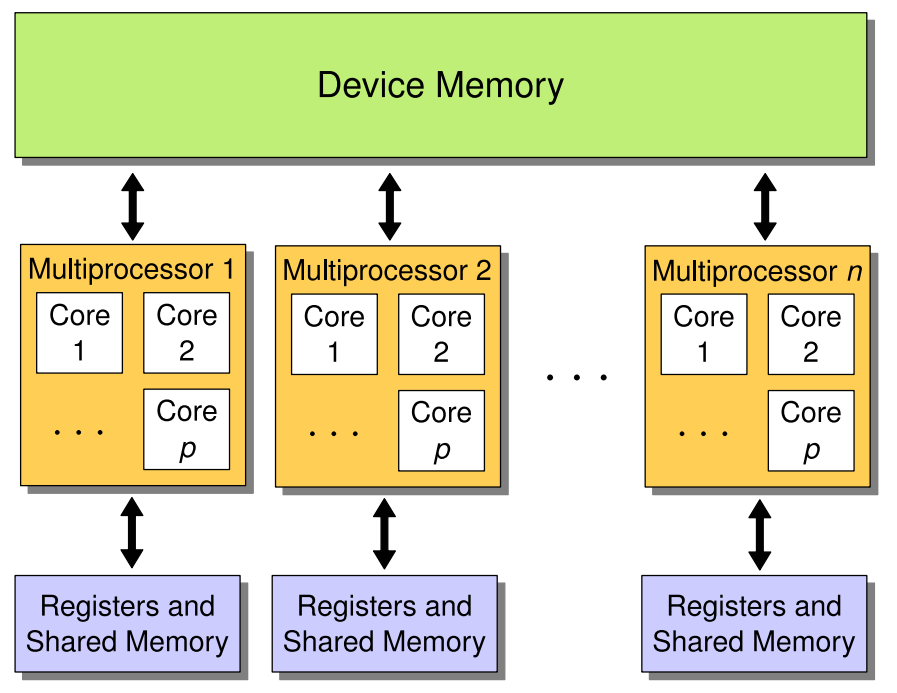

Figure 1: Architecture of a modern Nvidia graphics card. In Nvidia's nomenclature, cores are called stream processors (or scalar processors), and in current GPUs each multiprocessor has eight such cores.

280, paralleled in the HPC line by the Tesla C870 and C1060 (variants are also available in a single rack-mountable unit containing multiple GPUs). See Table 1 for detailed specifications. In Section 7 below, we benchmark our code on both the Tesla C870 and GeForce GTX 280. The Tesla C1060 was not available for benchmarking at the time of the conference.

A modern Nvidia GPU contains many multiprocessors, each composed of several cores, as illustrated in Fig. 1. For example, the GPU in the Tesla C1060 contains 30 multiprocessors and a total of 240 cores. Within a multiprocessor, cores are allocated local registers and have access to a fast shared memory. In addition, each multiprocessor provides two small read-only caches: a constant cache, and a texture cache to speed up global device reads. Primary storage on the card is provided by device memory, which is shared among all multiprocessors and has a relatively high latency. However, this latency can often be hidden by having a large number of threads ready to execute. A very important consideration is that the highest bandwidth from device memory is achieved when accesses are coalesced; this occurs when groups of 16 threads access a contiguous, properly aligned memory region. ${ }^{2}$

\footnotetext{
${ }^{2}$ This requirement has been relaxed somewhat in the more recent generation of cards.
} 


\section{The CUDA programming model}

The CUDA platform provides direct access to the GPU through a C-like programming language with minimal extensions. The CUDA platform includes a compiler that targets the GPU, as well as a hardware driver and a runtime library. Higher level libraries are also provided, including optimized BLAS and FFT implementations.

A CUDA application works by spawning a very large number of threads, as many as tens of thousands at once, which execute in parallel. For example, in a LGT application one might assign one thread to each lattice site. The user specifies the number, organization, and shared memory usage of the threads when a CUDA kernel is invoked. As an example, consider the CUDA code

$$
\text { dslashKernel <<<gridDim, blockDim, sharedBytes>>> (args); }
$$

which invokes dslashKernel (args) for execution by many individual threads on the GPU. Threads are grouped into thread blocks, and the entire collection of thread blocks is called a grid. The code above tells the GPU to launch a kernel using gridDim blocks, each containing blockDim threads. The compiler is instructed to allocate sharedBytes bytes of shared memory to each block. This shared memory allows for rapid communication between threads within a thread block. CUDA provides primitives to allow synchronization between threads within a thread block. However, no synchronization is possible between different thread blocks (within a single kernel invocation).

The GPU will dynamically schedule the thread blocks for execution. In order to hide high latency operations, it is desirable to have a high multiprocessor occupancy: each multiprocessor should have many threads simultaneously loaded and waiting for execution. The challenges to achieving high multiprocessor occupancy will be discussed in Section 6 . The GPU supports conditional execution, but it is highly desirable that groups of 32 threads (a thread warp) follow the same execution path. Otherwise, both execution paths are serialized and executed by the entire warp.

\section{Wilson inverter: Main features}

We implemented a CUDA kernel, Dslash, which calculates the Wilson-Dirac operator restricted to spinor variables at the even or odd parity sites. We have also implemented an even-odd preconditioned conjugate gradient $(\mathrm{CG})$ solver.

The action of Dslash and Dslash ${ }^{\dagger}$ on a spinor field is the most compute-intensive part of the CG inverter. Our CUDA implementation spawns one thread for each site in the (even or odd) sub-lattice. Performance is limited by memory bandwidth, as is common in LGT applications. Nonetheless, the GPU achieves a high sustained performance due to the relatively large memory bandwidth. We have structured the calculation in a manner which helps reduce the amount of transferred data (e.g., we mostly use 32-bit precision, we gauge fix to the temporal gauge, we reconstruct the gauge links from only two columns of data, etc.) and maximizes the overlap of computation and communication.

The CG algorithm is decomposed into several operations, each of which runs as a separate CUDA kernel. Besides the Dslash operator, we also require a dot product operation and a variety of BLAS-like operations. The dot product involves a global sum, which is performed by the GPU 
in double precision using parallel reduction. In the Wilson-Dirac solver, the largest portion of compute time is spent in applying the Dslash and Dslash ${ }^{\dagger}$ operators. We find that the whole solver runs at well over 80 percent of the speed of just its Dslash component. We also emphasize that the operation of the Wilson-Dirac solver involves negligible data transfer between the device and host computer.

\section{Data layout}

We consider a lattice of $3+1$ dimensions, splitting the gauge and spinor fields into even and odd sub-lattices. With three colors and four spin components, spinor fields require 24 floats per lattice site to store. Gauge fields require 18 floats per link. Following [1], we employ a specialized data layout. We do so because, as we have mentioned, maximum bandwidth is obtained when 16 consecutive threads (a half warp) simultaneously read 16 primitive elements which are packed contiguously in device memory. The available primitive elements include structures of 1,2 , or 4 packed floats. Our testing indicates that, on current hardware, the best bandwidth is achieved using device reads through the texture cache, and using float 4 primitives. Spinor objects are composed of 24 floats, so we use 6 arrays of float 4 s to store the entire spinor field. In this way, consecutive threads can simultaneously access (nearly) consecutive elements from device memory. The gauge links are stored in 12 floats (before $\mathrm{SU}(3)$ reconstruction), requiring 3 arrays of float $4 \mathrm{~s}$.

Because the lattice sites are split by parity, and because of boundary effects, the half warp of 16 consecutive threads may access float 4 objects which are nearly, but not exactly, contiguous in memory. The texture cache mitigates the performance penalty of imperfect memory accesses.

\section{Local storage constraints}

Unlike CPUs, the GPU does not provide a large memory cache. Instead, the GPU provides a relatively small amount of fast shared memory, which is manually managed and shared between threads. Shared memory is orders of magnitude faster than device memory, so access to the latter must be minimized. For the Dslash operation, we have found that shared memory alone is not satisfactory for local data storage, and we therefore employ registers for data storage as well.

For hiding high latency operations it is important to have a high multiprocessor occupancy: many active threads should be simultaneously loaded and ready to execute. Our tests indicate that 192 active threads per multiprocessor is desirable and that performance rapidly degrades with fewer active threads. The multiprocessor occupancy is determined by the register and shared memory requirements of the CUDA kernel.

At the hardware level, a single GPU contains many multiprocessors (currently 16 or 30). Each multiprocessor has a fixed quantity of local storage (registers and shared memory) which is divided among many active threads. Nvidia's previous generation GPUs provide 8,192 registers and $16 \mathrm{~KB}$ of shared memory per multiprocessor, while in the more recent generation the number of registers has been increased to 16,384 .

What are the local storage requirements for each thread in the Dslash operation? Each thread must accumulate to a single output spinor, which is composed of 24 floats and should reside in local storage. In constructing the output spinor, the thread loops over all neighboring sites. In each 


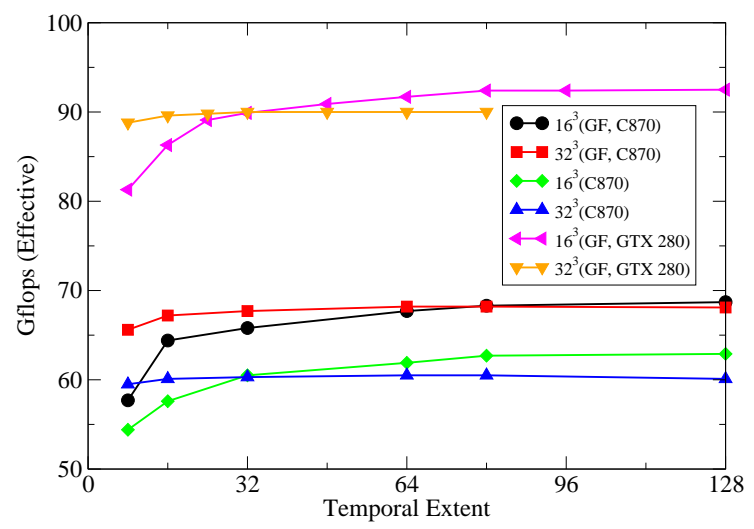

Figure 2: Performance of the preconditioned Wilson operator $\left(1-\kappa^{2} D_{e o} D_{o e}\right)$.

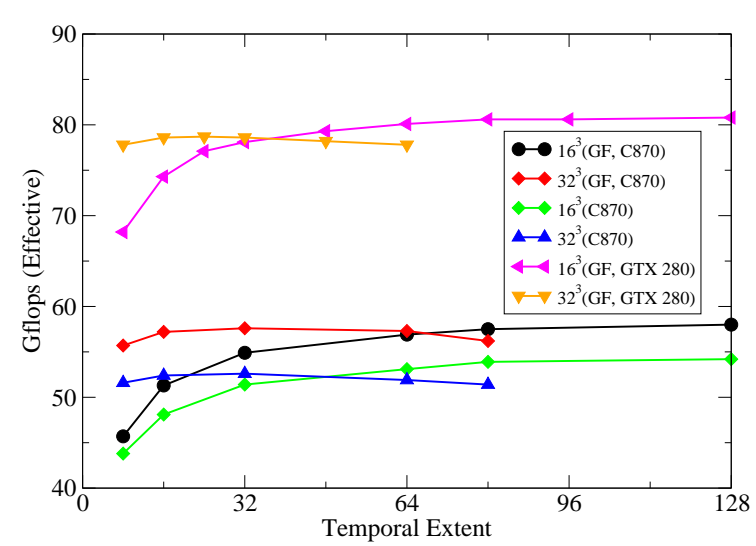

Figure 3: Performance of the conjugate gradient inverter.

direction, a full spinor and gauge link must be read. The neighboring spinor is immediately projected into a half spinor and requires only 12 floats of local storage. The SU(3) matrix representing the gauge link requires an additional 18 floats. Thus, at a minimum, 54 floats are required per thread. If these are to be stored entirely in the $16 \mathrm{~KB}$ of shared memory, then at most 64 threads would be active on one multiprocessor. ${ }^{3}$ This number is much smaller than the target, 192, and would negatively impact performance. Our trick is to use registers for additional data storage. The GPU has at least 8,192 registers per multiprocessor, providing $32 \mathrm{~KB}$ of local storage. Using both shared memory and registers it is possible to obtain 192 active threads per multiprocessor for the Dslash kernel.

What are registers exactly? Registers serve the same function on a GPU as they do on a CPU. Namely, they appear as explicitly labeled operands in machine code instructions generated by the compiler. Every active thread on a multiprocessor is allocated a sufficient number of private registers to execute the CUDA kernel. Unlike shared memory, registers cannot be shared between threads. Another limitation is that data stored in registers cannot be organized into an array and dynamically indexed. For example, we store the SU(3) matrix elements in registers by declaring

float g1, g2, g3, ..., g18;

We cannot use loops to express matrix operations on these elements. Writing the full Dslash operation by hand, and without using loops, would be tedious and error-prone. For this reason, we found it expedient to automatically generate the lengthy Dslash CUDA code. The Dslash code generator was written in the Scala programming language.

\section{Performance}

In Fig. Z, we present performance results for the even-odd preconditioned Wilson-Dirac operator on a range of different volumes. We find that the performance is only weakly volume-dependent: for all but the smallest volumes it sustains above 60 GFlops on the C870 and around 90 GFlops on

\footnotetext{
${ }^{3}$ The number of active threads must be a multiple of 32 , the warp size. A multiple of 64 is recommended.
} 
the GTX 280. Results for the full CG inverter are shown in Fig. 百. For volumes of reasonable size, the inverter sustains over 50 GFlops and 80 GFlops on the C870 and GTX 280, respectively. We have also implemented a BiCGstab inverter that achieves similar performance.

The (GF) label on the plots signify that the gauge-fixing trick was used. This involves fixing the temporal gauge links to the identity (on all but one time-slice) in order to save memory bandwidth, and improves performance by about 10 percent. We also save bandwidth by reading only two columns of each gauge matrix and reconstructing the third on the fly. In all cases, the reported performance numbers are "effective gigaflops" that may be compared with implementations on traditional architectures. In particular, the nominal number of operations per lattice site does not include the extra work done in the SU(3) reconstruction, nor the savings associated with having trivial links in the time direction.

We note that performance of the GPU code is more than an order of magnitude greater than typical SSE-optimized implementations (which generally achieve less than 5 Gflops for Wilson matrix-vector on a $3.0 \mathrm{GHz}$ quad-core Xeon processor). In addition, the scaling of performance with volume is relatively constant, as compared to CPU implementations which suffer dramatically as the local volume falls out of cache. At 80 Gflops sustained CG performance and $\$ 450$ per board, the GTX 280 card represents a price-performance ratio of $\$ 5.60 /$ Gflops (excluding, of course, the cost of the host computer).

\section{Acknowledgments}

This work was supported in part by US DOE grants DE-FG02-91ER40676 and DE-FC0206ER41440 and NSF grants DGE-0221680, PHY-0427646, and OCI-0749300.

\section{References}

[1] G. I. Egri, Z. Fodor, C. Hoelbling, S. D. Katz, D. Nogradi and K. K. Szabo, "Lattice QCD as a video game," Comput. Phys. Commun. 177, 631 (2007) [arXiv:hep-lat/0611022].

[2] K. Ibrahim, F. Bodin and O. Pène, "Fine-grained parallelization of lattice QCD kernel routine on GPUs," Journal of Parallel and Distributed Computing 68, 1350 (2008).

[3] NVIDIA Corporation, CUDA Programming Guide (2008), http://www.nvidia.com/object/cuda_develop.html

[4] Advanced Micro Devices, Inc., AMD Stream Computing User Guide (2008), http://ati.amd.com/technology/streamcomputing/resources.html

[5] F. Belletti et al., "QCD on the Cell Broadband Engine," PoS LAT2007, 039 (2007) [arXiv:0710.2442 [hep-lat]].

[6] J. Spray, J. Hill and A. Trew, "Performance of a Lattice Quantum Chromodynamics Kernel on the Cell Processor," Comput. Phys. Commun. 179, 642 (2008) [arXiv:0804.3654 [hep-lat]].

[7] H. Baier et al., "Status of the QPACE Project," arXiv:0810.1559 [hep-lat].

[8] L. Seiler et al., "Larrabee: a many-core x86 architecture for visual computing," ACM SIGGRAPH 2008, 1-15 (2008).

[9] A. Munshi, "OpenCL: Parallel computing on the GPU and CPU," presentation at SIGGRAPH 2008, http://s08.idav.ucdavis.edu/munshi-opencl.pdf 